\title{
Concept and framework of a self-regulating symbiotic network
}

\author{
David Plets ${ }^{1 *}$, Wout Joseph ${ }^{1}$, Eli De Poorter ${ }^{2}$, Luc Martens ${ }^{1}$ and Ingrid Moerman²
}

\begin{abstract}
The concept and framework of a self-regulating symbiotic network planner is introduced as a way to improve the use of available resources and infrastructure and the overall performance of co-located wireless networks. A framework for physical-layer optimization is proposed, based on an advanced and reliable network planner. Besides an optimal network planning including the adjustment of transmit powers, also a symbiotic optimization over different networks and network layers is implemented, a new concept in network cooperation based on shared and variable incentives. In this article, specifically, it is assumed that the co-located networks share the incentive of a lower global power consumption and the newly created symbiotic network is optimized accordingly. Feedback about the signal quality parameters allows optimizing path loss models, finetuning device transmit powers, coping with a changing propagation environment, and improving network reliability. The concept is applied to and experimentally validated with a real-life wireless test environment and a power consumption reduction of $79.5 \%$ is obtained, by consecutively enabling energy-saving features of the network planner: intelligent cognitive network planning, symbiotic network cooperation, and transmit power adjustments.
\end{abstract}

Keywords: Symbiotic, Network, Framework, Self-regulating, Sensor networks, Sensors, Optimization, Energy consumption, Power consumption, Reduction, Green

\section{Introduction}

In the recent years, an increasing number of networks using different wireless technologies started to co-exist: GSM (Global System for Mobile Communications), UMTS (Universal Mobile Telecommunications System), Bluetooth, WiMAX (Worldwide Interoperability for Microwave Access), Zigbee, DECT (Digital Enhanced Cordless Telecommunications), Wi-Fi, LTE (Long-Term Evolution), etc. The increase in the density of wireless devices, each using their own technology and having their own limitations (battery lifetime, memory capacity, etc.) leads to an increasing amount of interference and a sub-optimal use of available network resources, such as capacity.

In this article, the concept, the creation, and the framework of a self-regulating symbiotic network planner are developed and experimentally applied to an actual test network. The network planner automatically designs a

\footnotetext{
*Correspondence: david.plets@intec.ugent.be

1 Department of Information Technology, WiCa-Ghent University/IBBT, Gaston Crommenlaan 8, B-9050 Ghent, Belgium

Full list of author information is available at the end of the article
}

symbiotic network out of a set of co-located-independent wireless networks, based on a common incentive between the networks. Symbiotic networks [1] are coexisting homogeneous (using the same technology, e.g., two independent Wi-Fi networks from different companies, sharing their access points) or heterogeneous (e.g., Wi-Fi and UMTS networks allowing a smartphone user to collect data) networks that cooperate based on common incentives through infrastructure and resource sharing. Incentives are network goals that should be improved in order for the network to consider cooperating with other networks [2], e.g., a lower power consumption. The optimization is performed for multiple networks and over multiple network layers.

Even though a number of planning tools are able to predict network behavior of multiple network layers [3], these tools rely on accurate predictions of the network environment and cannot be used in dynamically changing networks. To overcome these shortcomings, the proposed solution introduces a feedback loop between the planning tool and the actual deployed network. By monitoring the

\section{量 Springer}

(c) 2012 Plets et al: licensee Springer. This is an Open Access article distributed under the terms of the Creative Commons Attribution License (http://creativecommons.org/licenses/by/2.0), which permits unrestricted use, distribution, and reproduction in any medium, provided the original work is properly cited. 
network, real-time network and signal quality information can be fed back into the planning tool to increase the accuracy of the used propagation models, to finetune transmit powers, or to adapt to a varying propagation environment or varying network conditions, as is the case when node failures occur. This allows an incentive-based optimization of the transmission settings. Also, the network planner can automatically be reconfigured when the shared incentives change. For example, in emergency cases, the network could decide to switch from minimal energy consumption to highly reliable communication.

The novelty of this article lies in the combination of different aspects: we have developed a network planner and optimizer for the physical and networking layer, in which intelligent cognitive reasoning methods are applied. The network planner implements three energy-saving features: (i) intelligent cognitive network planning, (ii) symbiotic network cooperation, and (iii) transmit power adjustments. To the best of the authors' knowledge, this is the first article to introduce the implementation of the symbiotic networking concept and a cognitive loop into an advanced and accurate physical-layer-based network planner for indoor environments. Moreover, as an application, the concept is applied to a real-life wireless test environment with actual measurements, where we aim to lower the power consumption without affecting coverage, by, e.g., minimizing the number of sink nodes.

The outline of this article is as follows. In Section 2, a short overview on related research on energy efficiency in sensor networks and the use of cognitive approaches is presented. Section 3 defines symbiotic networks, while in Section 4, the concept and benefits of self-regulating symbiotic network planning are discussed. Section 5 presents results of the application of the optimization concept to a real-life testbed network, and finally, conclusions are presented in Section 6.

\section{Related study}

A lot of research has already been performed in the field of energy consumption reduction in wireless sensor networks (WSNs), due to the many cases where network and device lifetime is of the utmost importance (e.g., for implanted devices in Wireless Body Area Networks [4]). Unlike in this article however, optimizations are usually performed at the Medium Access Control (MAC) layer, instead of at the physical layer. Also, optimization is often focused on one specific aspect of the network.

Different approaches have been followed in developing energy-efficient MAC protocols. As is stated in [5], two energy-saving approaches can be found in the literature: duty cycling and in-network aggregation. Adjustable duty cycling schemes for lower energy consumption are presented and simulated in [6,7] discusses an energyefficient MAC protocol based on ultra-low duty-cycle frame exchanges and scalable network self-configuration. The aggregation approach is mainly aimed at lowering the number of transmissions by using smart routing techniques, e.g., based on game theory [8]. In [9], an energybalancing routing scheme is proposed for longer network lifetimes. An analytical study of the deployment of trafficaware relay nodes is presented in [10]. In [11], an energy model for clustered multi-hop WSNs is derived and optimized with respect to the cluster-head selection process, hereby using a probabilistic method. A cluster-head is a node which collects data from other nodes and transfers it to the sink.

In [12], the authors have developed algorithms to minimize and balance energy consumption in WSNs with uniformly distributed sensor nodes, based on a sectorbased multi-hop approach. In [13], schemes are proposed that can lead to energy savings up to $30-70 \%$, by defining a cost function that also takes into account possible retransmissions. Zhang et al. [14] have focused on a realistic nonlinear battery model in a general two-hop relay network and developed the relay selection criterion from a battery energy efficiency perspective by following a theoretical and numerical approach.

Several papers perform a joint optimization on multiple layers: in both $[15,16]$, network lifetime is optimized on physical, MAC, and routing layers. The optimizations follow a theoretical-mathematical approach for a network with a single sink. An analytical approach for the determination of the optimal (common) transmit power in WSNs is presented in [17]. The influence of the exponent of the path loss model on the optimal transmit power is investigated, but just as in $[15,16]$, isotropic (one-slope) path loss models are assumed, which are often far from realistic in indoor environments with many different walls and wall types. Nonetheless, the correctness of these predictions is of major importance for the eventual performance of the proposed algorithms. In our research, an advanced indoor path loss model is used as the basis for all calculations. It takes into account the physical building layout and has been tested and validated in different indoor environments. Also, unlike in $[15,16]$, our optimization considers a topology with multiple sinks.

While cognitive approaches have already widely been applied to WSNs, they are usually used for spectrumsensing purposes $[18,19]$, rather than for signal quality feedback, which is done in this article. Spectrum sensing is the process of recording the occupation of the different frequency bands and the variation of this occupation over time. Knowledge of the (un)occupied frequency bands allows choosing the frequency band that is most suited for packet transmission. A holistic approach to cognition and a framework that can help achieve end-to-end goals of application-specific sensor networks is provided in [20]. In [21], models and algorithms for self-optimization are 
presented, in networks that are interconnected through a broadband wireless mesh backbone network. An architectural approach is followed, where positions of backbone and terminal nodes are optimized for very large networks (e.g., $50 \times 50 \mathrm{~km}^{2}$ ). The self-optimization is based on a mathematical model and uses flocking algorithms and particle swarm optimization, a relatively slow-converging optimization method.

Very often, the proposed (mechanism for) power consumption reduction is only considered in a theoretical or analytical way, or is based on simulations. For example, in [22] a new metric for energy-efficient cooperative transmission is introduced and applied to a theoretical case. Simulations of an energy-efficient clustering algorithm are presented in [23]. In this article however, the framework is experimentally tested in an actual network.

Not much research has been conducted on symbiotic networking. In [24], cooperation of heterogeneous networks is investigated at the level of a vertical handoff mechanism, based on a cross-layer polynomial regression predictive received signal strength (RSS) approach. A symbiotic integration of heterogeneous wireless networks at application-level is proposed in [25], while [26] discusses cooperative multicast on the physical layer, applied to heterogeneous networks. To the best of the authors' knowledge, this article is the first to combine the symbiotic networking aspect with a self-regulating physicallayer optimization.

To summarize, we can state that the existing literature on energy consumption reduction in WSNs mainly focuses on theoretical and analytical approaches and simulations. Also, in most of the articles, the optimization is performed on MAC or routing layers instead of on the physical-layer. In research that does take into account physical-layer aspects, the authors use very simple (and less accurate) isotropic path loss models, which are often unreliable in indoor environments. In this article, a framework for physical-layer optimization on multiple levels is proposed, based on an advanced and reliable network planner. Besides an optimal network planning including adaptable transmit powers, also a symbiotic optimization over different networks and network layers is implemented, a new concept in network cooperation. Moreover, a cognitive loop is added to the system, allowing self-regulation of the network planning process, improving network reliability, and adapting to varying propagation environments. Finally, our research does not only rely on theoretical calculations, but also an actual implementation of the optimization is experimentally tested in a wireless test network, increasing the contribution of this study. In the presented framework, advanced (energy) optimization strategies can be implemented and the framework can be used in cooperation with energy-efficient MAC protocols.

\section{Symbiotic networks}

Symbiotic networks [1] form a promising concept in a world where a lot of wireless technologies coexist independently from each other. They support cooperative networking over all layers between different nodes in possibly even different networks, in order to obtain even better networking optimizations. The aim of the cooperation is to provide benefits for all participating networks. Possible incentives to support cooperation between the different networks are decreased energy consumption, lower exposure, a longer device life time, lower interference, a higher throughput, a better quality of service, etc. These incentives can vary in the course of time.

However, while striving to meet each one of these incentives, it is crucial that the network remains operational, even when, e.g., transmit powers are lowered for the purpose of a lower energy consumption or when the propagation environment changes. Therefore, we choose to implement the symbiotic networking concept into a self-regulating indoor network planning tool.

Symbiotic networks are divided into two categories: homogeneous and heterogeneous symbiotic networks. A homogeneous symbiotic network is a network where different networks using the same technology cooperate. Examples are WiFi networks of different companies sharing their access points, different sensor networks sharing their sinks, etc. A heterogeneous symbiotic network is a network where different networks using different technologies cooperate. Since Bluetooth, Zigbee, and WiFi networks operate in the same frequency band, they could form a symbiotic network and cooperate for interference minimization by means of channel selection or time slot allocations. Another example is a mobile phone data access case where there is a joint planning of $3 \mathrm{G} / 4 \mathrm{G}$ femtocells and WiFi access points.

\section{Self-regulating symbiotic network planning}

This article aims to create a symbiotic physical-layer optimization of different wireless networks, which is automatically controlled by means of a self-regulating (cognitive) feedback loop. These functionalities are implemented in a previously developed network planner (WiCa Heuristic Indoor Propagation Prediction-WHIPP [27,28]). In this section, we will first go into the WHIPP network planner, followed by a description of the practical implementation of the self-regulating cognitive mechanism into this network planner. Finally, the advantages of the self-regulating symbiotic approach are highlighted.

\subsection{Indoor network planner}

The heuristic planning algorithm (WHIPP) has been developed and validated for the prediction of path loss in indoor environments $[27,28]$. It takes into account the effect of the environment on the wireless propagation 
channel and has been developed for the prediction of the path loss in zones of about $5 \mathrm{~m}^{2}$ or at specific locations. It bases its calculations on the determination of the dominant path between transmitter and receiver, i.e., the path along which the signal encounters the lowest obstruction. This approach is justified by the fact that more than $95 \%$ of the energy received is contained in only two or three paths [29]. The dominant path is determined with a multidimensional optimization algorithm that searches the lowest total path loss, consisting of a distance loss (taking into account the length of the propagation path), a cumulated wall loss (taking into account the walls penetrated along the propagation path), and an interaction loss (taking into account the propagation direction changes of the path, e.g., around corners). The model has been constructed for the $2.4 \mathrm{GHz}$ band and its performance has been validated with a large set of measurements in various buildings [27]. In contrary to many existing tools no tuning of the tools parameters is performed for the validation. Excellent correspondence between measurements and predictions is obtained, even for other buildings and floors [27]. As our tool is based on the free-space loss model for every environment, the tool is generally applicable, while other tools are often too dependent of the environment upon which the used propagation model is based. The network planner is also able to calculate the optimal access point placement for a given user-defined coverage.

\subsection{Network planner functionalities for test network}

In this section, the different functionalities of the network planner will be illustrated: optimal sink selection, establishing symbiotic cooperation between networks, setting and optimizing node parameters, and path loss model optimization.

First, the network planner (logically) has the capability of optimizing wireless networks and is thus able to derive the number and location of the sinks (i.e., energy-consuming nodes that collect the data from the other nodes) using the coverage optimization algorithm described in [27]. For two independent sensor networks that have the common incentive 'lower energy consumption', the symbiotic network planner could, e.g., decide to reduce the total number of sinks, without affecting coverage. Second, the network planner is also able to establish a symbiotic cooperation between the different co-located networks. This cooperation has extensively been discussed in [30]. Third, the network planner is able to set the nodes' transmit/receiver mode on or off and to control the nodes' transmit power. And fourth, the network planner can also tune its propagation models for more reliable predictions and hence better future decisions. These four features will be illustrated in Section 5, where they will be applied to a real-life wireless test network.

\subsection{Cognition implementation}

Tuning of the propagation models and optimization of the node parameters is done based on a feedback loop. This self-regulating network planning process is illustrated in Figure 1. The three building blocks of Figure 1 (network planner, network, and database) and their connection will now be explained.

The process begins with the symbiotic network planning algorithm [27] calculating the optimal node parameters. This calculation depends on which of the network planning features (see above) are enabled, as well as on the agreed common incentives between the networks. Once the optimal node parameters (sinks, transmit powers, modes, etc.) have been determined, the network (Figure 1) is reconfigured in a second step and packets are sent accordingly. To this end, a java tool is developed that can send configuration messages (radio on, radio of, set tx power, etc.) to the sensor devices. For the experiments, the configuration messages were sent over wired debugging interfaces, but the concepts can also be used when configuration messages are sent over the wireless interface. Information consistency between a node and its sink is ensured by relying on reliable networking protocols on higher layers (e.g., the Zigbee protocol).

To determine whether or not the network behaves as expected, link quality information (received signal strength indicator-RSSI, average noise floor, etc.) is

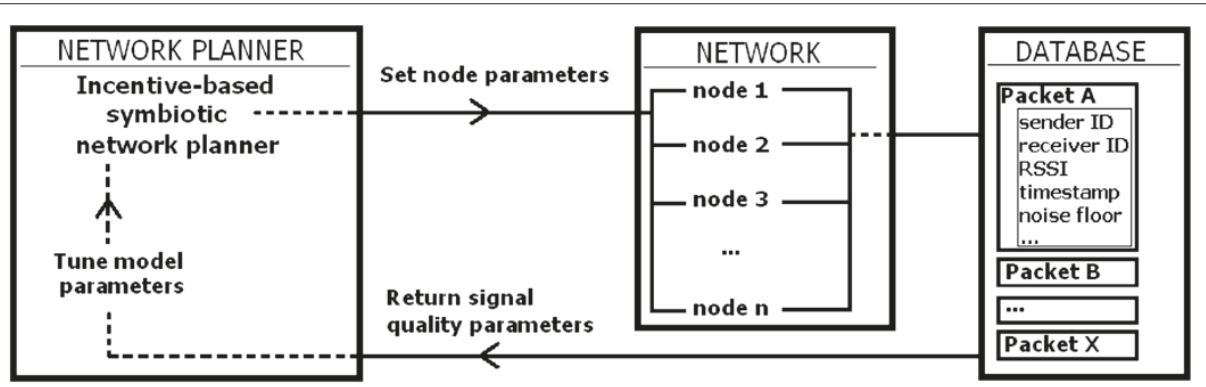

Figure 1 Flow graph of interaction between self-regulating symbiotic network planner and network. 
collected by the sinks. In case of multi-hop networks, intermediate receiver nodes will transfer the link quality information to the sink. For experiments, the same wired debugging interface is then used. However, even when this data are collected over the wireless interface, the overhead will be very low since (i) the configuration data are typically minimal (e.g., one message every hour) compared to the data traffic from the sensor network and (ii) network monitoring and configuration is only needed at the beginning of the network deployment or when the wireless environment changes significantly. In fact, once the cognitive planning tool finalizes optimizing the network, only changes to the network environment that last a long time need to be communicated. As such, after the initial configuration, the monitoring overhead becomes insignificant compared to the operational network energy consumption.

Then, on a central PC, the measured data are stored in an SQL database (Figure 1) as input for the cognitive planning tool. To collect the information gathered by the sinks at a central control PC, each sink device is capable of communication with the central control PC to store the gathered information. In our experimental proof-of-concept implementation, the sinks and the control PC are connected using a wired ethernet connection, but the concepts can equally be applied to networks where sink devices use (long range) wireless communication technologies to exchange information between each other.

The use of a symbiotic network planner requires additional network structure in the form of a central server that is capable of collecting all the measured information and is able to control the settings of the wireless nodes. The network planner can be run as a software component on a server or, alternatively, the optimization process can be calculated by an independent (external) server that gains access to the collected data. Existing current-day management platforms are already capable of controlling the settings of wireless nodes in a building, for example, to support handovers between different access points in wireless conferencing systems. Our symbiotic network planner extends these or similar systems with the capability to fulfill specific network incentives.

\subsection{Advantages}

In view of meeting the agreed incentives while still keeping the network operational, a self-regulating feature is implemented into the network planner: signal quality data are returned from the database to the network planner and used to recalculate the optimal network settings. With respect to energy consumption, node lifetime, and network robustness, several advantages are associated to a self-regulating network planning. First, based on the observed RSSI values, the used path loss models can be tuned to cope with prediction inaccuracies (feedback loop in Figure 1). This allows a more reliable estimation of the connection quality and possibly, an optimization of the transmit power of the nodes (increased node lifetime). Second, the self-regulating process is able to automatically deal with changes in the physical network layout. This for example means that depending on the specific case and on the network topology, it allows detecting node failures and recovering from it. Also, our symbiotic planning tool allows coping with a varying propagation environment. This could, e.g., be the case in museums, where a temperature or humidity sensor is attached to the paintings. A rearrangement of the aisles in the exhibition hall would then not require a manual adjustment of the network parameters, but it could be dealt with by the self-regulating network planner.

\section{Application of self-regulating symbiotic network planner to real-life wireless test network}

As a proof-of-concept, the self-regulating symbiotic network planner is applied to a real-life wireless test network. In a first section, this test network is described. Then, the actual application of the network planner to this network is discussed.

\subsection{Wireless test network}

The application of the network planner is executed within the w-iLab.t test network [31,32]. It is an experimental generic wireless testbed for development and testing of wireless applications via an intuitive web-based interface. The architecture of the testbed is based on the widely used MoteLab testbed concept from Harvard University. Registered users can upload executables, associate those

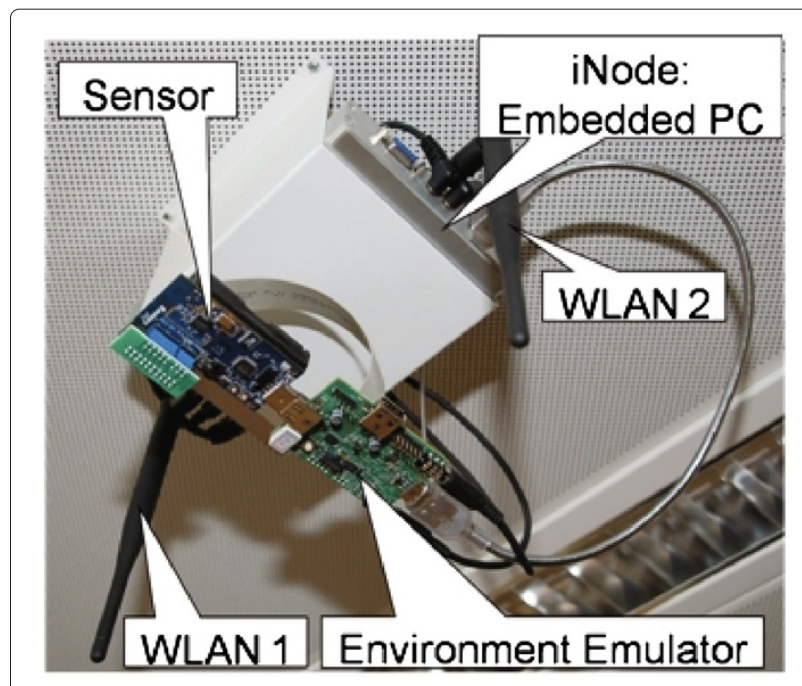

Figure 2 w-iLab.t node with indication of WiFi interfaces, sensor node, embedded pc, and environment emulator. 


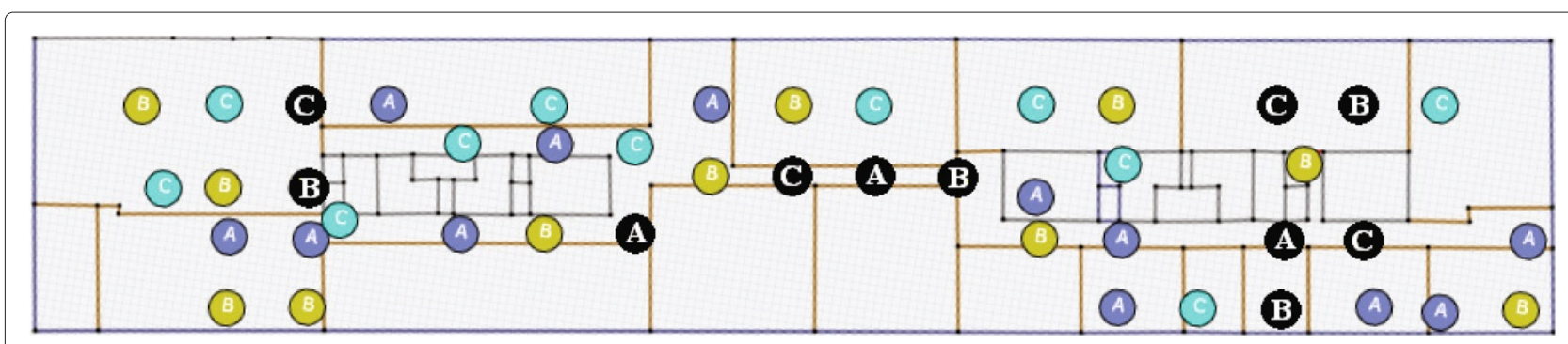

Figure 3 Original test network nodes from three networks (A, B, C) (phase 1) and their corresponding (optimal) sinks (black) (phase 2).

executables with motes to create a job, and schedule the job to be run on the w-iLab.t network. The nodes and their settings can also be controlled by a Java program, as is the case for this research. During the job all messages and other data are logged to a database which is presented to the user upon job completion and then can be used for processing and visualization.

In total, 45 nodes, equipped with 2 Wi-Fi IEEE $802.11 \mathrm{a} / \mathrm{b} / \mathrm{g}$ wireless network interfaces (type COMPEX WLM54-SAG23) and 1 or 2 TMoteSky sensor nodes with IEEE 802.15.4 interface embedded with temperature, light, and humidity sensors, have been installed at a height of $2.5 \mathrm{~m}$ on the third floor of an office building in Ghent, Belgium. The nodes (i-Nodes) are embedded PCs equipped with Ethernet, USB, etc. The sensor chip is an radio frequency (RF) transceiver designed for low-power and low-voltage wireless applications and has a programmable output power, varying in eight steps between -25 and $0 \mathrm{dBm}$. In receiving mode, the RSSI indicates the received power and is a good indicator for the packet reception rate when the noise is limited [33]. Figure 2 shows one of the 45 nodes, with the indication of the WiFi antennas and the sensor node. Figure 3 shows the location of all the (operational) nodes of this wireless test network on the third floor of the office building in which the w-iLab.t network is deployed.

\subsection{Application of self-regulating symbiotic network planner}

The network planner is here applied for a lower global power consumption (incentive). In four consecutive phases, different features of the network planning tool will be enabled in order to reduce the total power consumption.

1. Initially (phase 1, Table 1), all 45 nodes are 'on', each of which consumes $65.01 \mathrm{~mW}$, yielding a resulting total power consumption of $2925.5 \mathrm{~mW}$ (see Figure 4). The nodes belong to three different sensor networks, indicated with a different color and a different letter inside the marker (A, B, or C, see Figure 3). This is considered as the first of four phases between which subsequent energy reduction optimizations are executed. Table 1 summarizes the characteristics of the different phases in the power consumption optimization process.

2. After applying the network planner as a first optimization step (phase 2, see Table 1), the sinks for each network are determined, and they are marked with black dots (see Figure 3). To be able to receive data from other nodes, the radio of the sink node should always be active and is therefore the main contributor to the total energy consumption, provided the data traffic in the network is limited. The 11 sinks together thus consume $11 \cdot 65.01 \mathrm{~mW}=715.1 \mathrm{~mW}$. The other 34 nodes are assumed to be 'on' during, e.g., $20 \%$ of the time, to allow sending packets and detecting incoming packets. The value of $20 \%$ will depend on the type of network and the intended communication. Under this assumption, the power consumption of these 34 nodes is $34 \cdot 65.01 \mathrm{~mW} \cdot 20 \%=442.1 \mathrm{~mW}$, resulting

Table 1 Different phases in the power consumption optimization process and their characteristics $(Y=y e s, N=n o)$

\begin{tabular}{|c|c|c|c|c|c|c|}
\hline & \multicolumn{3}{|c|}{ Use of } & \multirow{2}{*}{$\begin{array}{c}\text { Number } \\
\text { of } \\
\text { sinks }\end{array}$} & \multirow[b]{2}{*}{$\begin{array}{c}\text { Power } \\
\text { consumption (mW) }\end{array}$} & \multirow[b]{2}{*}{$\begin{array}{c}\text { Reduction } \\
\text { (\%) }\end{array}$} \\
\hline & $\begin{array}{l}\text { network } \\
\text { planner }\end{array}$ & $\begin{array}{c}\text { symbiotic } \\
\text { cooperation }\end{array}$ & $\begin{array}{c}\text { transmit } \\
\text { power control }\end{array}$ & & & \\
\hline Phase 1: basic network & $\mathrm{N}$ & N & $\mathrm{N}$ & - & 2925.5 & - \\
\hline Phase 2: network planner & Y & N & $\mathrm{N}$ & 11 & 1157.2 & 60.5 \\
\hline Phase 3: symbiotic & Y & Y & $\mathrm{N}$ & 4 & 793.1 & 72.9 \\
\hline Phase 4: transmit power & Y & Y & Y & 4 & 600.5 & 79.5 \\
\hline
\end{tabular}




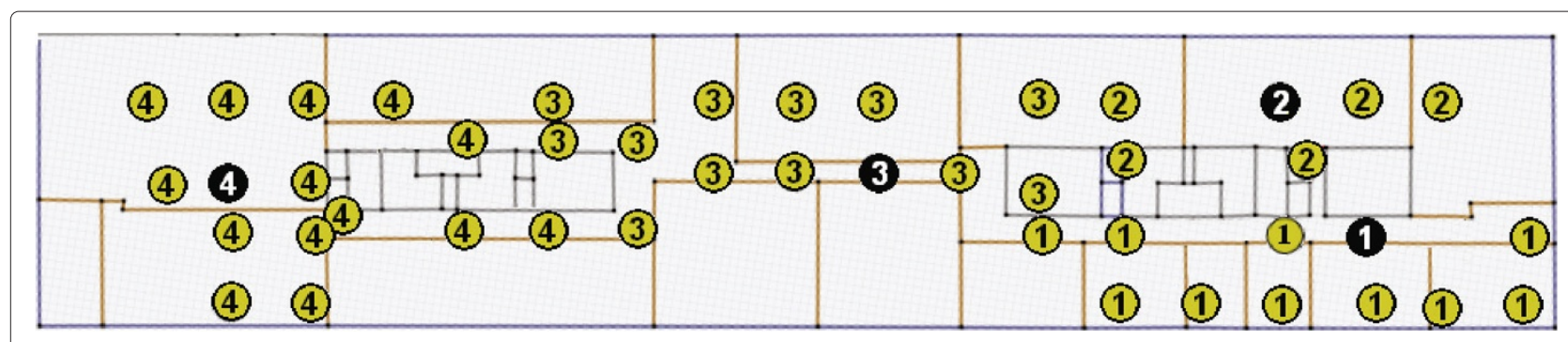

Figure $4 \mathrm{Global}$ network power consumption in the different optimization phases.

in a total energy consumption of $1157.2 \mathrm{~mW}$ for all 45 nodes (see Figure 4).

After selecting these sinks, 100 packets are sent by each node to its corresponding sink and signal quality data are logged in a database (Figure 1). The feedback loop from the database towards the symbiotic network planner then allows the adjustment of the prediction models, based on the difference between the RSSI recorded at the receiver nodes and the RSSI predicted by the path loss model. Figure 1 illustrates how this process is repeated. A first comparison between the measured path loss $\mathrm{PL}_{\text {measured }}$ and the predicted path loss $\mathrm{PL}_{\text {predicted }}$ in the symbiotic network yielded an average prediction error $\delta=\mathrm{PL}_{\text {measured }}-\mathrm{PL}_{\text {predicted }}$ of $-3.9 \mathrm{~dB}$ and an average absolute prediction error $|\delta|$ of $6.8 \mathrm{~dB}$. Applying the symbiotic network planner and adapting the path loss model with this fixed offset of $-3.9 \mathrm{~dB}$ results in a prediction improvement of $1.9 \mathrm{~dB}$, with an average absolute error $|\delta|$ of $4.9 \mathrm{~dB}$ in the second run. Although this article only intends to present a framework into which more advanced

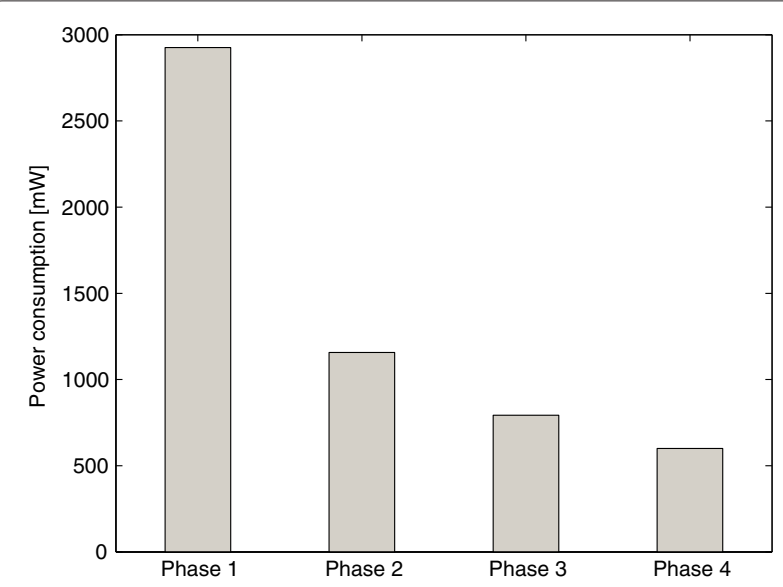

Figure 5 Symbiotic network after application of the symbiotic network planner (sinks indicated with black dot and number, other nodes send to sink with corresponding number $(1,2$, or 3$)$ ) (phases 3 and 4). strategies can easily be implemented, either for adjusting the path loss models or for the incentive-based symbiotic network planner, the improvement of $1.9 \mathrm{~dB}$ already indicates the usefulness of the feedback loop (e.g., for finetuning the nodes' transmit power, see phase 4).

3. In the third phase (symbiotic phase, see Table 1), symbiotic cooperation between the networks is introduced (see Table 1). The common incentive for these three networks is now to consume less energy. Based on sharing the infrastructure of the three networks, the incentive-based network planner first determines the minimal number of required sinks and their optimal location for an operational symbiotic network [27]. The planning tool uses its internal path loss models (which were finetuned in the previous phase) and the node characteristics to predict how many sinks are needed to be able to reach a sink from each of the nodes, and where these sinks should be located. Figure 5 shows the resulting symbiotic network, containing only four sinks instead of eight for the original network (Figure 3). The independent networks $\mathrm{A}, \mathrm{B}$, and $\mathrm{C}$ cooperate and now form one symbiotic network, where each of the nodes sends its data to the corresponding sink (black dots in Figure 5): the numbers in the nodes indicate which sink the respective nodes send their data to (nodes with marker $i$ send to sink $i, i=1,2,3,4$ ). The sink can either be a node from the sending node's original network, or a node from another network. The symbiotic network has a total power consumption of $4 \cdot 65.01 \mathrm{~mW}$ (sinks) $+41 \cdot 65.01 \mathrm{~mW}$ . $20 \%$ (other 41 nodes) $=793.1 \mathrm{~mW}$ (see Figure 4).

Table 2 Sensor power consumption for different RF transmit powers

\begin{tabular}{lc}
\hline RF power $(\mathbf{d B m})$ & Sensor power consumption $(\mathbf{m W})$ \\
\hline 0 & 57.4 \\
-5 & 46.2 \\
-10 & 36.3 \\
-15 & 32.7 \\
\hline
\end{tabular}


4. The cognitive loop not only allows the tool to improve its network planning models, the feedback about the actual path loss values also allows a further optimization of the global energy consumption (phase 4 in Table 1). In this phase, the transmit powers in the symbiotic network are individually optimized for each sensor node. Knowledge of the receiver sensitivity and the link path loss fed back by the cognitive loop, allows calculating the minimally required node transmit power. Transmission power control mechanisms have already been implemented in some wireless communication protocols. A centralized physical-layer solution can however optimize the power consumption and minimize interference. According to the obtained values, 8 nodes require a transmit power of $0 \mathrm{dBm}, 9$ require a power of $-5 \mathrm{dBm}$, and 24 nodes require a power of $-10 \mathrm{dBm}$ or $-15 \mathrm{dBm}$ (12 each). Table 2 shows the sensor power consumption for four different transmit powers. With the assumed duty cycle of $20 \%$, this leads to a total power consumption of $4 \cdot 65.01 \mathrm{~mW}+(8 \cdot 57.4 \mathrm{~mW}+9 \cdot 46.2 \mathrm{~mW}+12$. $36.3 \mathrm{~mW}+12 \cdot 32.7 \mathrm{~mW}) \cdot 20 \%=600.5 \mathrm{~mW}$ (see Figure 4). Table 1 and Figure 4 show that the subsequent optimization phases enable a total energy consumption reduction of almost $80 \%$ for the network under test. As a baseline for the power consumption reduction, we have chosen phase 1 (without any network planning intelligence), because we believe it is still common practice that sensor networks are installed with every node in 'always active'-mode. However, when assuming phase 2 (after network planning) as the baseline, we still obtain reduction percentages of 31.5 and $48.1 \%$ for the symbiotic optimization (phase 3 ) and the transmit power optimization (phase 4), respectively.

\section{Conclusions}

The concept, creation, and framework of an advanced physical-layer-based self-regulating symbiotic network planner are presented as a way to improve the overall performance of co-located wireless networks. The planning tool creates an optimized incentive-based symbiotic network starting from different independent wireless networks. Optimization is performed for multiple networks and over multiple network layers in a real-life testbed sensor network, based on actual measurements and for the shared incentive of a lower global power consumption of the co-located networks. Besides an optimal network planning including the adjustment of transmit powers, also a symbiotic optimization over different networks and network layers is implemented. Feedback about the signal quality parameters is used for optimization of the path loss models and for finetuning device transmit powers. The framework is also experimentally applied to an actual wireless test network. The use of the network planner first reduces the total energy consumption from 2926 to $1157 \mathrm{~mW}$. Feedback about the measured signal quality produced a prediction improvement of $1.9 \mathrm{~dB}$ after a single model adjustment when applying the planner to a wireless test network example. With the improved prediction models and after enabling symbiotic cooperation, the global power consumption further reduces to $793 \mathrm{~mW}$. Finally, the cognitive loop allows finetuning the nodes' transmit powers, leading to a further power consumption reduction to $601 \mathrm{~mW}$. The total reduction equals $80 \%$ for the network under test. In the presented framework, more advanced (energy) optimization strategies can be implemented and the framework can be used in cooperation with energy-efficient MAC protocols. In the future, also other incentives (e.g., the lowest exposure) can be investigated.

\section{Competing interests}

The authors declare that they have no competing interests.

\section{Acknowledgements}

This study was supported by the IWT-SBO SymbioNets project. W. Joseph and E. De Poorter are Post-Doctoral Fellows of the FWO-V (Research

Foundation-Flanders).

\section{Author details}

${ }^{1}$ Department of Information Technology, WiCa-Ghent University/IBBT, Gaston Crommenlaan 8, B-9050 Ghent, Belgium. ² Department of Information Technology, IBCN-Ghent University/IBBT, Gaston Crommenlaan 8, B-9050 Ghent, Belgium.

Received: 16 March 2012 Accepted: 22 October 2012

Published: 14 November 2012

\section{References}

1. ED Poorter, B Latre, I Moerman, P Demeester, Symbiotic networks: towards a new level of cooperation between wireless networks. (Special Issue) Wirel. Personal Commun. J. 45(4), 479-495 (2008)

2. ED Poorter, P Becue, I Moerman, P Demeester, in IEEE International Conference on Communications (ICC 2011). Exploring a boundary-less cooperation approach for heterogeneous co-located networks, Kyoto, Japan, 2011)

3. Y Wu, P Chou, Q Zhang, K Jain, W Zhu, S-Y Kung, Network planning in wireless ad hoc networks: a cross-layer approach. IEEE J. Sel. Areas Commun. 23(1), 136-150 (2005)

4. E Reusens, W Joseph, B Latre, B Braem, G Vermeeren, E Tanghe, L Martens, I Moerman, C Blondia, Characterization of on-body communication channel and energy efficient topology design for wireless body area networks. IEEE Trans. Inf. Technol. Biomed. 13(6), 933-945 (2009)

5. G Campobello, A Leonardi, S Palazzo, Improving energy saving and reliability in wireless sensor networks using a simple crt-based packet-forwarding solution. IEEE/ACM Trans. Netw. 20(1), 191-205 (2012)

6. H Yoo, M Shim, D Kim, Dynamic duty-cycle scheduling schemes for energy-harvesting wireless sensor networks. IEEE Commun. Lett. 16(2), 202-204 (2012)

7. M Kohvakka, J Suhonen, TD Hamalainen, M Hannikainen, Energy-efficient reservation-based medium access control protocol for wireless sensor networks. EURASIP J. Wirel. Commun. Netw. 2010 (article ID 878412) (2010). http://jwcn.eurasipjournals.com/content/2010/1/878412

8. S Mehta, KS Kwak, An energy-efficient MAC protocol in wireless sensor networks: a game theoretic approach. EURASIP J. Wirel. Commun. Netw. 
2010(article ID 926420) (2010). http://jwcn.eurasipjournals.com/content/ 2010/1/926420

9. H Zhang, H Shen, Balancing energy consumption to maximize network lifetime in data-gathering sensor networks. IEEE Trans. Parallel Distrib. Syst. 20(10), 1526-1539 (2009)

10. F Wang, D Wang, J Liu, in 6th Annual IEEE Communications Society Conference on Sensor, Mesh and Ad Hoc Communications and Networks, 2009. SECON '09. Traffic-aware relay node deployment for data collection in wireless sensor networks, Rome, Italy, June 2009), pp. 1-9

11. J Choi, C Lee, Energy consumption and lifetime analysis in clustered multi-hop wireless sensor networks using the probabilistic cluster-head selection method. EURASIP J. Wirel. Commun. Netw (2011). http://jwcn. eurasipjournals.com/content/2011/1/156

12. M Nickray, A Afzali-Kusha, R Jantti, MEA an energy efficient algorithm for dense sector-based wireless sensor networks. EURASIP J. Wirel. Commun. Netw (2012). http://jwcn.eurasipjournals.com/content/2012/1/85/ abstract

13. S Banerjee, A Misra, in Proceedings of the 3rd ACM International Symposium on Mobile Ad Hoc Networking \& Computing, ser. MobiHoc. Minimum energy paths for reliable communication in multi-hop wireless networks (ACM, New York, NY, 2002), pp. 146-156. http://doi.acm.org/10.1145/513800. 513818

14. W Zhang, D Duan, L Yang, in IEEE Military Communications Conference, 2009. MILCOM 2009. Relay selection from a battery energy efficiency perspective, Boston, Massachusetts, USA, October 2009), pp. 1-7

15. H Wang, N Agoulmine, M Ma, Y Jin, Network lifetime optimization in wireless sensor networks. IEEE J. Sel. Areas Commun.

28(7), 1127-1137 (2010)

16. R Madan, S Cui, S Lall, A Goldsmith, in IEEE Proceedings of 24th Annual Joint Conference of the IEEE Computer and Communications Societies, INFOCOM 2005, vol. 3. Cross-layer design for lifetime maximization in interference-limited wireless sensor networks (USA, Miami, Florida, March 2005), pp. 1964-1975

17. S Panichpapiboon, G Ferrari, O Tonguz, Optimal transmit power in wireless sensor networks. IEEE Trans. Mobile Comput. 5(10), 1432-1447 (2006)

18. T Yucek, H Arslan, A survey of spectrum sensing algorithms for cognitive radio applications. IEEE Commun. Surv. Tutor. 11(1), 116-130 (2009)

19. G Ganesan, $Y \mathrm{Li}$, in First IEEE International Symposium on New Frontiers in Dynamic Spectrum Access Networks, 2005. DySPAN 2005. Cooperative spectrum sensing in cognitive radio networks (Baltimore, Maryland, USA, November, 2005), pp. 137-143

20. G Vijay, E Ben Ali Bdira, M Ibnkahla, Cognition in wireless sensor networks: a perspective. IEEE Sensors J. 11(3), 582-592 (2011)

21. H Zhang, J Llorca, C Davis, S Milner, Nature-inspired self-organization, control, and optimization in heterogeneous wireless networks. IEEE Trans. Mobile Comput. 11(7), 1207-1222 (2012)

22. B Li, W Wang, Q Yin, R Yang, Y Li, C Wang, A new cooperative transmission metric in wireless sensor networks to minimize energy consumption per unit transmit distance. IEEE Commun. Lett. 16(5), 626-629 (2012)

23. D Wei, Y Jin, S Vural, K Moessner, R Tafazolli, An energy-efficient clustering solution for wireless sensor networks. IEEE Trans. Wirel. Commun. 10(11), 3973-3983 (2011)

24. B-J Chang, J-F Chen, Cross-layer-based adaptive vertical handoff with predictive rss in heterogeneous wireless networks. IEEE Trans. Veh Technol. 57(6), 3679-3692 (2008)

25. Y-C Lai, P Lin, S-M Cheng, Performance modeling for application-level integration of heterogeneous wireless networks. IEEE Trans. Veh. Technol. 58(5), 2426-2434 (2009)

26. X Xie, B Rong, T Zhang, W Lei, Improving physical layer multicast by cooperative communications in heterogeneous networks. IEEE Wirel. Commun. 18(3), 58-63 (2011)

27. D Plets, W Joseph, K Vanhecke, E Tanghe, L Martens, Coverage prediction and optimization algorithms for indoor environments. EURASIP J. Wirel. Commun. Netw. (Special Issue on Radio Propagation, Channel Modeling, and Wireless, Channel Simulation Tools for Heterogeneous Networking Evaluation . 1 (2012). http://jwcn.eurasipjournals.com/content/2012/1/ 123

28. D Plets, W Joseph, K Vanhecke, E Tanghe, L Martens, Simple indoor path loss prediction algorithm and validation in living lab setting. Wirel Personal Commun, 1-18 (December 2011). doi:10.1007/s11277-011-0467-4. http:// dx.doi.org/10.1007/s11277-011-0467-4
29. G Wølfle, R Wahl, P Wertz, P Wildbolz, F Landstorfer, in German Microwave Conference (GeM/C). Dominant path prediction model for indoor scenarios, Ulm, Germany, April 2005)

30. ED Poorter, P Becue, M Rovcanin, I Moerman, A negotiation-based networking methodology to enable cooperation across heterogeneous co-located networks

31. IBBT, Ibbt - w-ilab.t. http://wilab.atlantis.ugent.be/

32. CREW, Crew project-cognitive radio experimentation world. http:// www.crew-project.eu/wilabt/

33. K Srinivasan, P Levis, RSS/ is under-appreciated, (Cambridge, MA, May 2006)

doi:10.1186/1687-1499-2012-340

Cite this article as: Plets et al.: Concept and framework of a self-regulating symbiotic network. EURASIP Journal on Wireless Communications and Networking 2012 2012:340

\section{Submit your manuscript to a SpringerOpen ${ }^{\circ}$ journal and benefit from:}

- Convenient online submission

- Rigorous peer review

- Immediate publication on acceptance

- Open access: articles freely available online

- High visibility within the field

- Retaining the copyright to your article

Submit your next manuscript at $\boldsymbol{\triangleright}$ springeropen.com 